\title{
Concepciones de los estudiantes universitarios sobre la naturaleza de la ciencia/conocimiento científico desde las perspectivas epistemológicas empirista/positivista, constructivista y crítico social
}

High school student's perceptions on science foundations and scientific knowledge base on an epistemological empirist and positivist approach and on a constructivist and critical social perspective

Roberto Figueroa Molina, Ed.D.

Profesor Universidad del Attántico. Barranquilla-Colombia. Director Grupo "GECT". robertofigueroamolina@gmail.com, roberfigue@hotmail.com

\section{Resumen}

Esta investigación se enmarca en el área de los estudios científicos de las ciencias de la educación. Describe las concepciones de los estudiantes universitarios sobre la naturaleza de la ciencia y el conocimiento/cient́fico desde las perspectivas epistemológicas empirista/positivista, constructivistas y critico social. Es decir, determina las implicaciones que tienen estas tendencias conceptuales en las estructuras curriculares de los programas académicos de las instituciones universitarias.

Los resultados de este estudio proveen información significativa sobre los alumnos en cuanto al trasfondo filosófico-metodológico de la ciencia, que puede ser utilizado por instituciones educativas de manera efectiva, en el desarrollo de currículos, estrategias de enseñanza y otras aplicaciones encaminadas a fortalecer el conocimiento y el entendimiento sobre la naturaleza de la ciencia y el conocimiento científico.

\section{Palabras claves}

Concepciones, naturaleza de la ciencia, conocimiento cientúfico, perspectivas epistemológicas empirista/positivista, constructivista y crítico social.

\section{Abstract}

This Work is within the scientific studies of educational science. It describes the high school student's perceptions on science foundations and scientific knowledge, based on an epistemological/empinist and positivist approach an on a constructivist and critical social perspective. In other words, it establishes the implications related to these conceptual trends within the curricular structures of the academic programs within high school institutions.

The condusions of this study provide significative information about how science philosophical and methodological background can be used effectively by educational institutions to develop consistent educational curricula teaching strategies and then applications that allow to strengthening the knowledge and understanding of science foundations and scientific knowledge.

\section{Keywords}

Perceptions, Science foundation, scientific knowledge, epistemological empirist and positivist, constructivist and critical social. 


\section{Introducción}

Las crecientes investigaciones sobre las concepciones que tiene los estudiantes sobre el conocimiento cient́fico, han evidenciado problemas en la adquisición, asimilación y construcción de marcos conceptuales en los procesos de enseñanza y aprendizaje de las ciencias (Posner, Strike, Hewson y Gertzog, 1982; Osbome y Freyberg, 1985; Driver, 1985), producto del conocimiento subyacente que poseen los estudiantes con respecto a los fenómenos universales. A pesar de la necesidad de identificar en nuestros estudiantes y maestros las concepciones que dificultan el aprendizaje de las ciencias, al momento del presente estudio no se encontraron evidencia sobre instrumentos o estrategias pedagógicas que investigadores nacionales o extranjeros hayan desarrollado y validado, que logren caracterizar la aultura e idiosincrasia de los estudiantes y profesores en el campo de las ciencias.

La naturaleza de la ciencia es uno de los elementos conceptuales de la cultura científica, que la educación en ciencia de diferentes países como Estados Unidos, Inglaterra, Francia, Alemania, Finlandia y otros, deben desarrollar. Para que los estudiantes y maestros en formación adquieran un conjunto de conocimientos y de destrezas de pensamiento, que le permitan relacionarse con un mundo natural, para reconocer su diversidad y su unidad. La comprensión de los conceptos fundamentales, los principios de la ciencia, la metodología, las matemáticas y la tecnología como empresa humana son parte de la cultura científica.

Sin embargo, aun en estos países conociendo la importancia de la naturaleza de la ciencia/conocimiento científico, es poco el énfasis que se le ha dado al tema dentro de la educación cientúfica (Spector, 1996; Ortiz, 1987). Aspectos como el dominio de contenido por los maestros (Cleminson, 1990), y la escasez de elementos filosóficos y epistemológicos sobre la naturaleza de la ciencia/conocimiento científico (Hodson, 1986; Stenhause, 1985; Duschl, 1988; Ambibola, 1983), contribuyen para que las concepciones epistemológicas no estén daras y sean resistentes al cambio.

Es necesario pues, distinguir entre las concepciones epistemológicas de la ciencia y la simple carencia de información sobre éstas. En las primeras, los estudiantes responden con bastante convencimiento, mediante determinados tipos de razonamientos donde utilizan unas ideas y relaciones determinadas que le lleven a cometer ciertas equivocaciones "típicas" y no otras. En el segundo caso, las contestaciones serían más bien en blanco o aleatorias (Nussbaum y Novick, 1982).

Se suelen admitir, sin reticencias, la existencia de concepciones de los estudiantes (Duit, 1981; Erickson, 1980), acerca de asuntos con los que están más familiarizados, pero hay quienes se resisten a aceptar que esos estudiantes tengan concepciones más o menos elaboradas sobre contenidos académicos (Sen'ko, 1983). Las concepciones que tienen los estudiantes acerca de los diversos aspectos de las materias escolares difieren, pues, significativamente, de los contenidos de los programas escolares, con el agravante de que, además, suelen estar muy arraigadas en el individuo y ser muy resistente al cambio.

Dentro de las actuales posibilidades de indagar adecuadamente qué conocimiento tienen los estudiantes que ingresan a la universidad, sobre la naturaleza de la ciencia/conocimiento cient́fico, surge la necesidad de construir instrumentos o estrategias educativas, que permitan identificar hasta que punto los estudiantes están inmersos en el quehacer científico y cómo éstos pueden relacionar las actividades educativas y sociales de su entomo con los procesos de la ciencia. Para esto es necesario inquirir sobre los nuevos planteamientos curriculares que el sistema educativo ha propuesto sobre la naturaleza de la ciencia cómo uno de los elementos básicos de la cultura científica y hasta qué punto los estudiantes y los docentes, han participado en el aprendizaje y enseñanza de estos aspectos de la ciencia. 
Por lo tanto, con la introducción de elementos epistemológicos en la enseñanza de las ciencias, puede establecerse como objetivo, que los estudiantes aprendan de manera comprensiva los conocimientos científicos que se consideran apropiados para cada edad. Estos principios epistemológicos deben estar en los programas de enseñanza, o bien pueden proponerse como finalidad utilizar estos conocimientos como herramientas para desarrollar destrezas de pensamiento (Villarini, 1992). La introducción de estos elementos epistemológicos tiene por intención presentar la ciencia de manera holística no constituida por partes aisladas que aparecen aquí y allá sin ninguna relación dara entre sí. Por el contrario, debemos contemplarla como constituida por eslabones de una cadena continua de reflexiones de aciertos y errores, tan característicos los unos como los otros para el avance del pensamiento que construyen los científicos (Moreno, 1986).

El Science for American-Project 2061 (1989), manifiesta que la cultura cientúfica induye, además de las ciencias, matemáticas y tecnología, el poseer una base filosófica sobre la ciencia. Sabemos que no se trata simplemente de intentar que el estudiante reproduzca el mismo proceso seguido por los científicos a lo largo de la historia. Entre otras muchas razones porque los científicos no reprodujeron ningún proceso seguido previamente por otros, y este hecho de por sí sólo, introduce ya una diferencia tan fundamental entre lo que se supone es la realidad que hace totalmente imposible este intento.

El aprendizaje sobre la naturaleza de la ciencia/conocimiento científico debe capacitar al estudiante, para enfrentarse a los avances tecnológicos, a la manipulación y al uso inacional de químicos y muchas otras situaciones que demandan el conocimiento de destrezas de pensamiento relacionados con la ciencia. Cada vez es más imprescindible que las personas posean estos aspectos básicos de la ciencia para que comprendan los fenómenos naturales y tecnológicos a los que estará expuesto por siempre (Bonett, 1991).

Aunque es necesario este conocimiento, no podemos negar la existencia de concepciones epistemológicas erróneas en cualquier dominio cient́fico y concretamente en el campo de la ciencia, aparece hoy suficientemente probada (Carrascosa, 1987). Existen, no obstante, serias discrepancias respecto a la efectividad de una enseñanza que tenga en cuenta dichas concepciones (Hewson y Hewson, 1984; Fredette y Lockhead, 1980). Queremos mostrar entonces, que los estudiantes tienen concepciones, a menudo sólidamente integradas como evidencia de sentido común, y ello tiene lugar no sólo en estudiantes de niveles elementales, sino que también afecta a estudiantes universitarios e incluso una parte del profesorado.

La existencia de dichas concepciones está íntimamente ligada a una metodología que lleva a dar respuestas "seguras" y rápidas, que son consecuencia de generalizadiones acríticas de observaciones cualitativas. Esta metodología de lo aparente que se traduce en certeza, en ausencia de dudas o de consideraciones de posible soluciones altemativas está profundamente arraigada, por responder a las formas de atención y reflexión cotidianas habituales de los individuos (Piaget, 1971).

La cultura científica es una dimensión del conocimiento que toda persona educada debe conocer, para responder efectivamente a los retos y problemas que nos presenta la realidad, como ciudadanos responsable e inteligente y como consumidores informados (Williams, 1992). El desarrollo de destrezas asociadas a la cultura científica requiere de metas daras y modelos no tradicionales para la enseñanza de la ciencia que utilice el inquirir, la exploración, la curiosidad natural de los seres humanos y el trabajo cooperativo entre otros. 


\section{Desarrollo}

Según Collette y Chiappetta (1986), un atributo distintivo de un individuo que posee cultura científica es que éste posea una concepción adecuada de la naturaleza de la ciencia. Los educadores de ciencia se han relacionado con el término Naturaleza de la Ciencia por más de tres décadas, definiéndolo de varias formas. Para Lederman (1987), la definición del término Naturaleza de la Ciencia más común es la siguiente: se refiere a las hipótesis, valores y suposiciones inherentes al desarrollo del conocimiento cient́fico. Por ejemplo un individuo que piense que el conocimiento cient́fico es amoral, tentativo, empírico o producto de la creatividad humana está reflejando su concepción individual sobre la naturaleza de la ciencia. En 1951 James B. Conant, señalo que no es suficiente estar informado sobre la ciencia, sino más bien entender la naturaleza de esta disaiplina (Cobem, 1989). Para Cobem, lo que es necesario entender de la naturaleza de la ciencia son sus suposiciones, sus métodos, sus estructuras y sus limitaciones.

Muchos científicos, educadores y filósofos de la ciencia han planteado que las ciencias son mucho más que una colección de hechos, conceptos y principios. (Lederman (1987) plantea que la naturaleza de la ciencia se caracteriza por los siguientes atributos: el conocimiento cient́fico es tentativo, histórico, público, empírico, probalístico, limitado, establece relaciones de causa y efecto y no puede responder a todas las preguntas del ser humano.

En la actualidad se hace imprescindible que el maestro conozca y comprenda los atributos de la naturaleza y el quehacer científico (Lederman, 1987). Según este autor, no se puede esperar que el maestro enseñe lo que no comprende. Por otro lado Duchl (1985) enfatiza la importancia que tiene para la educación en ciencia que el maestro tenga el dominio de la naturaleza de la ciencia. También señalan que el conocimiento del quehacer científico es fundamental en la sociedad actual. El conocimiento que tengan los individuos de una sociedad sobre la ciencia dependerá en gran medida de las experiencias provistas por los maestros de ciencia en el salón de dase. Por eso, es de suma importancia que el maestro comprenda la naturaleza de la ciencia para que pueda hacer un modelaje y una representación genuina de ésta en el salón de dases. La noción que tengan los docentes sobre la naturaleza de la ciencia indudablemente, influenciará a los estudiantes en la comprensión de esta disciplina; es decir, el docente de ciencia tiene que comprender la naturaleza de la ciencia porque es responsabilidad transmitir ese conocimiento a sus estudiantes.

El docente de ciencia debe ser capaz de comprender las fortalezas y limitaciones del quehacer cientúfico. En el salón de dase se debe crear un balance entre el énfasis que ofrezca a la ciencia como un banco de conocimiento, como proceso y como una empresa humana. El profesor debe estar daro en la estructura del conocimiento cientúfico y cómo éste se desarrolla de manera que se pueda garantizar de una forma genuina un conocimiento cient́fico legitimo. En otras palabras el educador en ciencia debe conocer la naturaleza de esta disciplina para proveer una enseñanza autentica.

\section{Método}

En este estudio se utilizó un diseño con un enfoque de naturaleza descriptiva. El propósito fue describir las concepciones sobre la naturaleza de la ciencia/conocimiento científico desde la perspectiva epistemológica empirista/positivista, constructivista y crítico social, de los estudiantes universitarios de ciencia experimental de la Universidad del Atántico. Esto se realizó partiendo de un instrumento previamente validado con estudiantes universitarios (Figueroa 1997). Además, se investigó que relación había entre las concepciones epistemológicas de los estudiantes y su preferencia por la ciencia y se determinó sí existían diferencias estadística significativa en la perspectiva epistemológica empirista/positivista, constructivista y cútico social en función de las 
variables: género, área de concentración o facultad, ubicación geográfica de la escuela donde estudio y el tipo de escuela donde estudió.

En este estudio se utilizaron estadísticas descriptivas como la media aritmética, por ciento, frecuencia y desviación estándar que sirvieron para describir las variables de la investigación. El análisis de varianza (ANOVA) para establecer las diferencias entre las submuestras con relación a la perspectiva epistemológica empirista/positivista, constructivista y crítico social, igualmente la prueba $t$ y la prueba delta. La prueba de Ji-auadrado para determinar diferencias significativas por género, facultad o concentración de estudio, el tipo de escuela donde estudió (pública-privada) y la ubicación geográfica de la escuela (zona rural o zona urbana) si se cumplen los supuestos de las mismas.

\section{Resultados}

Para responder que concepciones epistemológicas sobre la naturaleza de la ciencia/conocimiento cient́fico tienen los estudiantes se realizó una distribución de frecuencia, se determinó el por ciento, la media aritmética y la desviación estándar de los datos obtenidos de la muestra. Estos datos se analizaron de acuerdo con una escala que se estableció en tres categorías: "de acuerdo" "indeciso" y "en desacuerdo".

El análisis de los datos totales agrupados en las tres categorías muestran que para el Factor 1: Visión/tendencia empirista/positivista el $73.1 \%$ de los estudiantes respondieron estar "de acuerdo" con las afirmaciones de esta visión epistemológica, el $13 \%$ de los estudiantes con la categonía "indeciso" y el 15.7\% de los estudiantes con la categoría "en desacuerdo" de la misma visión. Para el Factor 2: Visión/tendencia constructivista, el $63.7 \%$ de los estudiantes contestaron estar "de acuerdo" con las afirmaciones constructivistas, el $23.6 \%$ de los estudiantes con la categoría "indeciso" y el 12.7\% de los estudiantes con la categoría "en desacuerdo" de la misma visión epistemológica. Para el Factor 3: Visión/tendencia crítico social, el $65.7 \%$ de los estudiantes contestaron estar "de acuerdo" con las afirmaciones cútico social, el $20.6 \%$ de los estudiantes con la categoría "indeciso" y el 13.7\% de los estudiantes con la categoría "en desacuerdo" de la misma visión epistemológica. Es decir, que todos los ítemes pertenecientes al Factores 1, Factor 2 y Factor 3 se contestaron dentro de la categoría "de acuerdo" y la categońa "indeciso", lo que se expresa como una homogeneidad en las respuestas de los estudiantes hacía a las alternativas totalmente de acuerdo, de acuerdo y no se qué decir. Estas respuestas muestran que los estudiantes de universitarios del área de ciencias experimentales de la universidad del atlántico, no tiene una concepción epistemológica sobre la naturaleza de la ciencia/conocimiento científica desde la perspectiva filosófica empinista/positivista, constructivista y crítico social.

Al comparar las medidas de tendencia central (media aritmética, la moda y la mediana), de los datos totales agrupados con relación a la variable concepciones epistemológicas de los estudiantes, se observa que tanto la puntuación de la moda como la de la mediana son iguales (3.00) y la puntuación de la media aritmética (2.985). Lo cual evidencia que no hubo una curva de distribución normal, ya que la tendencia de las respuestas se distribuye haća la categoría "de acuerdo".

Para determinar sí existía una relación entre las concepciones epistemológicas de los estudiantes participantes del estudio y su preferencia por la ciencia. Se introdujo en el instrumento en la sección relacionada con la información general, una pregunta sobre la ciencia con las alternativas: "Me gusta mucho" (5), "Me gusta" (4), "Me es indiferente" (3), "Me gusta poco (2) y "No me gusta" (1), en una escala de cinco puntos. Los resultados reflejan que el $20.3 \%$ de los estudiantes universitarios de ciencias experimentales de la universidad del atántico, le gusta mucho la ciencia, el $46.7 \%$ de los estudiantes le gusta la ciencia, para el $9.9 \%$ de los estudiantes le es indiferente, al $14.1 \%$ le gusta poco la ciencia y al $9.0 \%$ no le gusta la ciencia. 
Para dar respuesta a la pregunta de investigación, hacia cuál perspectiva epistemológica exhiben una tendencia los estudiantes, se realizó un análisis, que consistió en establecer la diferencia entre las contestaciones totales ofrecidas por cada estudiante en el Factor 1, el Factor 2 y Factor 3 (Factor1 - Factor 2 = ( $\Delta$ ) Delta) (Factor1 - Factor $3=(\Delta)$ Delta). (Factor2 - Factor $3=$ $(\Delta)$ Delta). Este análisis se realizó debido a que los datos obtenidos para determinar las concepciones epistemológicas de los estudiantes, no presentaban una distinción entre una concepción y la otra. La tendencia de responder más afirmativamente hacia la perspectiva epistemológicas empirista/positivista, no es resultado que los estudiantes hayan contestado de forma correcta los ítemes pertenecientes a ésta perspectiva epistemológica, sino el producto de establecer una diferencia entre el mayor número de veces que los estudiantes respondieron estar más "totalmente de acuerdo" y "de acuerdo" con respecto a una u otra perspectiva epistemológica.

Sin embargo, la diferencia que logra obtenerse sólo nos lleva a expresar que los estudiantes respondieron estar más veces "totalmente de acuerdo" y "de acuerdo" con la perspectiva epistemológica empirista/positivista que con la perspectiva epistemológica constructivista y crítico social. Es decir, que esta tendencia hacia la perspectiva epistemológica empirista/positivista no es producto de un razonamiento lógico, sino producto de la casualidad.

Para responder como varían las concepciones epistemológicas que tienen los estudiantes sobre la naturaleza de la ciencia/conocimiento científico de acuerdo al género, área de concentración o especialidad (Facultades de Ciencias Básicas (Biología, Química y Física) Ingenierías, Educación (Ciencias Naturales y Educación Ambiental), zona geográfica donde esta ubicada la escuela (ruralurbana) y el tipo de escuela donde estudió pública-privada (laica, católica, protestante) se llevó a cabo una prueba de Ji-cuadrado a un nivel de significación alfa de 0.05 , tabulación cruzada y un análisis de varianza (ANOVA). Los resultados obtenidos para la prueba Ji-cuadrado no permiten establecer diferencia estadística significativa en las concepciones epistemológicas de los estudiantes de primer año universitario con relación al género, área de concentración, zona geográfica donde esta ubicada la escuela y el tipo de escuela pública o privada. Los datos señalan que existe homogeneidad en las respuestas de los estudiantes. Esas se agrupan en las categorías "de acuerdo" e "indeciso", quedando vacía la celda de la categoría "en desacuerdo", por lo que no se cumple acabalidad con los supuestos de la misma.

También se llevó a cabo una prueba t, para establecer sí existía diferencia entre promedios al contrastar las puntuaciones de hombres y mujeres. La prueba $t$ refleja que no existe diferencia estadística significativa para la variable género y las perspectivas epistemológicas que tienen los estudiantes. Dos análisis de varianza (ANOVA) fueron desarrollados para establecer diferencias entre las variables área de concentración, tipo de escuela donde estudio y ubicación geográfica de la escuela, con relación a las concepciones epistemológicas que tienen los estudiantes. El análisis refiejan que los datos no cumplen con los supuestos de la prueba, y la misma no eran válida para determinar sí existía differencia estadística significativa.

\section{Discusión}

Los resultados obtenidos reflejan que los estudiantes universitarios de la ciencia experimentales de la universidad del atántico que respondieron la escala Likert, no tienen una concepción epistemológica sobre la naturaleza de la ciencia/conocimiento científico, pero sí una tendencia hacia la perspectiva epistemológica empirista/positivista. En este sentido Pérez-Gómez y Gimeno (1988), expresan que para entender el pensamiento y la actuación de los estudiantes, no basta con identificar los procesos formales y las estrategias de procesamiento de información o toma de decisiones, hay que calar en la red ideológica de teorías y creencias que determinen el modo de cómo los estudiantes dan sentido a su mundo general y a su contexto en particular. 
Paradójicamente, éste es el ámbito sobre el que se han realizado menos estudios dentro del enfoque cognitivo que estamos considerando.

Una de las razones que podemos aducir del porqué esta muestra de estudiantes no tienen una concepción epistemológica, puede deberse a que un número apreciable de los profesores de ciencia del nivel universitario de la universidad del atlántico, no tienen un dominio aceptable acerca de la naturaleza de la ciencia según Rivas (1997). Los resultados de este estudio coinciden con los informados por Méndez, Oquendo y Rivas (1996), quienes realizaron una investigación para determinar el conocimiento de los maestros de escuela elemental de la Región Educativa de San Juan Puerto Rico, acerca de la naturaleza de la ciencia. En dicha investigación se encontró que los profesores de ciencia del nivel elemental no poseen un conocimiento adecuado acerca de la naturaleza de la ciencia.

La homogeneidad en las respuestas de la muestra de estudiantes de aceptar de manera categórica todo tipo de afirmaciones, refleja cierto hilo conductor que tiende a adjudicar la misma importancia a la observación de la realidad frente a la especulación mental; lo tangible y concreto frente a lo abstracto, lo cierto y seguro frente a lo probable; y en definitiva lo real, práctico y objetivo, frente a lo meramente racional y teórico, por un lado y lo subjetivo y personal por otro. Esta homogeneidad en las respuestas de los estudiantes, la podemos asemejar con los períodos de ciencia normal Kuhn (1962), en los que los científicos guiados por un único paradigma incuestionado en sus aspectos básicos, resuelven los problemas teóricos o experimentales de su área mejorando la articulación y la coherencia interna del mismo. Este grupo de estudiantes, aunque no tiene una concepción epistemológica, están aceptando de manera categórica las afirmaciones que constituyen el instrumento y resolviendo de manera "segura" en ausencia de duda su posición filosófica. Esta tendencia a responder de manera rápida y "segura", es consecuencia de generalizaciones acríticas de observaciones aualitativas. Esta forma de contestar según Piaget (1971), se presenta como un hábito o "metodología", en donde la aparente se traduce en certeza en ausencia de duda o de consideraciones de posibles soluciones alternativas están profundamente arraigadas, para responder a las formas de atención y reflexión cotidianas habituales del niño.

Los estudiantes tienen concepciones sólidamente integradas como evidencia del sentido común, aceptando sin ninguna reflexión todas las afirmaciones que guarden una similitud conceptual con su corpus de conocimientos elaborados empíricamente. Según Carrascosa y Gil (1985), la existencia de dichas concepciones están íntimamente ligadas a una "metodología de la superficialidad" que lleva a dar respuestas rápidas, que son consecuencia de generalizaciones acríticas de observaciones cualitativas.

La tendencia de los estudiantes tanto a escala general como particular hacia la perspectiva empirista/positivista, estriba en el hecho que los estudiantes no poseen experiencias concretas que le sirvan de contraste con sus ideas. Sin embargo, los estudiantes han sufrido cierto proceso de homogeneización de sus creencias debido, en primer lugar, al hecho de disponer todos de un mismo ámbito de experiencias "la enseñanza" y, por tanto, de un mismo referente común. En segundo lugar y más importante, quizás, se debe a la asimilación cultural que realizan de su ambiente o entomo y de los estereotipos y prototipos que en él son dominantes. Otro factor que pudo influir en la determinación de esta visión/tendencia epistemológica es el relacionado con el área de concentración, ya que los mayores por ciento encontrados en el análisis de la diferencia "delta" provienen de aquella muestra de estudiantes que desarrollan estudios en las facultades de Ingeniería y carreras afines.

\section{Conclusiones}

A la luz de lo presentado se establecen las siguientes condusiones: 
1. Los estudiantes universitarios de los programas de ciencias experimentales no exhiben una concepción epistemológica definida sobre la naturaleza de la ciencia/conocimiento científico desde las perspectivas filosóficas empirista/positivista, constructivistas y critico social.

2. Aunque estudiantes universitarios de los programas de ciencias experimentales no tienen una concepción epistemológica acerca de la naturaleza de la ciencia/conocimiento cient́fico, exhiben una tendencia hacia la perspectiva epistemológica empirista/positivista.

3. Los estudiantes universitarios de los programas de ciencias experimentales les gusta la ciencia. Pero esta tendencia no evidencia que tengan una concepción epistemológica sobre la naturaleza de la ciencia/conocimiento científico.

4. La variable género no guarda relación con las concepciones epistemológicas que poseen los estudiantes que participaron del estudio. Tampoco existe diferencia estadística para la variable género y las perspectivas epistemológicas que exhiben los estudiantes.

5. Debido a la homogeneidad en las respuestas de los estudiantes hacia la alternativas "totalmente de acuerdo" y de "acuerdo", no hubo dispersión en la distribución de las puntuaciones, es decir, los datos obtenidos no cumplen con los supuestos de las pruebas, y las mismas no eran válidas para determinar si existía diferencia estadística en las perspectivas epistemológicas con relación a las variables; área de concentración o facultad ubicación geográfica de la escuela y el tipo de escuela donde estudió.

\section{Bibliografía}

Abimbola, I O. (1983). The revelence of the "new" phylosophy of science for the science curriculum. School Science and Mathemathics, 83 (3), 181-192

American Association for the Advancement of Science (1989). Science for Al/ Americans: Project 2061. Washington, D. C.

Bonnet, J.A. Jr., (1991). Nuevos conceptos para la enseñanza de las ciencias. Ponencia presentada en el Foro del Ateneo. Puertorriqueño sobre Ciencia y Tecnología. San Juan Puerto Rico.

Carrascosa, J. y Gil, D. (1985). Los errores conceptuales en la enseñanza de la Física II, Una propuesta para un tratamiento. Primeras Jomadas de Investigación y Renovación de la Física y Química en el BUP y COU. Valencia.

Carrascosa, J. (1987). Tratamiento didáctico en la enseñanza de las ciencias, de los errores conceptuales. Tesis doctoral, Facultad de Química de la Universidad de Valencia.

Cobem, W. W. (1990). A logic-structural, worldview analysis of the interrelationship between scence interest gender and concep of nature (ERIC Document No. ED 329426).

Cleminson, A. F. (1990). Establishing an epistemological base for science teaching in the light of contemporary notions of the nature of science and of how children leam science. Journal of Research in Science Teaching, 27 (5), 429-445.

Collette, A. Y Chiapptta, E. L (1986). Science Instruction in the middle and secondary schools. Columbus: Merill.

Driver, R. (1985). Beyond appearence the conservation of matter under physical and chemical tranformations. Children's Ideas in Saience, 10, 47-51.

Duit, R. (1981). Understanding energy as a conserved quantity. European Joumal of Science Education, 3 (3), 291.

Duschl, R. A. (1988). Abondoning the science legacy of science education. Science Education, 15, 129.

Eridkson,G. (1980). Childrens conception of heat. Science Education. 64,323-338. 
Figueroa, R, (1997). Desarrollo y validación de un instrumento para medir las concepciones sobre la naturaleza de la ciencia/conocimiento científico de los estudiantes de primer año universitario de Puerto Rico desde la visión/tendencia empirista/positivista y constructivista. Internado doctoral inédita, Universidad de Puerto Rico.

Fredette, N y Lochead, J. (1980). Students conception of electic current. The Physics Teacher, $18,83-90$.

Hewson, P. y Hewson M. (1984). The role of conceptual conflict change and the design of science instruction. Instructional Science, 13, 1-13.

Hodson, D. (1986). Philosophy of science and science curriculum. Joumal of Philosophy Education, 29, 215-225.

Kuhn, T. (1962). The structure of scientific knowledge University of Chicago Press. (Trad. Esp. La estructura de las revoluciones científica. Madrid: Fondo de Cultura Económica, 1986).

Lederman, N. G: (1992). Students and teachers conceptions of the nature of Science a review of research. Journal of Research Teaching, 28 (4), 331-359.

Nussbaum, J. y Novidk, S. (1982). Altemative frameworks, conceptual conflict and accommodation: Towards a principled teaching strategy. Instructional Science, 11, 183-200.

Ortiz, M. (1987). Ciencia ¿̇ara qué?. Prisma, Año II. (2), 107-123.

Osbome, R. y Freyberg, R. (1985). Learming science. Portsmouth, N. H.: Heinemann.

Pérez-Gómez, A. y Gimeno, J. (1988). Pensamiento y acción en el profesor: de los estudios sobre la planificación del pensamiento práctico. Infancia y Aprendizaje, 42, 37-63.

Piaget, J. (1971). Psicología y epistemología. Barcelona: Ariel.

Posner, G. J., Strike, K. A., Hewson, P. W., y Gertzog, W. A. (1982). Accomodation of scientific conception: Towards a theory of conceptual change. Saience Education, 66, 211-227.

Rivas, A. (1997), Conocimiento de los maestros de ciencia del nivel secundario de la región educativa de San Juan sobre la naturaleza de la ciencia. Tesis doctoral inédita, Universidad de Puerto Rico.

Sen'ko, R. U. (1983). Correcting the precientific conceptions of school children. Fizca V. Shokole. 1972. n。 1, Soviet Education Nov.-Dic, 1973.

Spector, P. E. (1996), Restructuring Science Rooms: A symbolic interaction study. Joumal of Research in Science Teaching, 33, 433-453.

Stenhouse, D. (1985). Active phylosophy in education and science Boston, M. A.: G. Allen \& Unwin Ltd.

Villarini, A. (1992). Manual para la enseñanza de destrezas de pensamienta. Proyecto de Educación Liberal Liberadora (PEL). San Juan, Puerto Rico.

Williams, J. (1992). On the Needs for Scientific Literacy Synergy. National Science Foundant. 